\title{
Transformation of heat into mechanical energy by means of rotating systems
}

\author{
Marian Mešina ${ }^{1, *}$ \\ ${ }^{1}$ Privat, D-73266 Bissingen, Mörikestraße 25, Germany
}

\begin{abstract}
All heat engines need two different temperatures for their work, $T_{1}<T_{2}$. The efficiency is limited by the Carnot formula from above. This article presents a new conception for heat engines. Unlike conventional heat engines, the working medium has an additional degree of freedom, the rotation around a given axis. The heat introduced and removed can not only lead to a change in the parameters of temperature, pressure, and volume, which are considered in conventional thermodynamics, but also to a change in the state of rotation. The rotational speed must also be taken into account in all phases of the cycle for all efficiency calculations. In many cases, this leads to a surprisingly different result from the results of conventional thermodynamics, that the efficiency of the cycle can exceed the Carnot limit. The efficiency values depend not only on temperatures and rotational speeds, but also on the material data. The proposed new type of heat engine makes it possible to better utilise very small temperature differences and under certain conditions, in combination with an ideal heat pump, to extract ambient heat and convert it into mechanical energy. The calculated results were presented for simple geometry and can easily be verified experimentally. In combination with an ideal heat pump, the proposed heat engine facilitates the surroundings to withdraw heat and convert it into mechanical work.
\end{abstract}

Keywords: Heat-mechanical energy conversion, efficiency, RS machine, Carnot

\section{Introduction}

In conventional thermodynamics, the maximum efficiency of heat engines can be fully specified by means of temperatures in the range of which the working cycle takes place. Carnot's theorem is the well-known formulation of this fact.

This article presents a whole new principle for converting heat into mechanical energy. The systems under consideration have an additional degree of freedom - the rotation around their own axis. The heat supplied can also partly be converted into the acceleration of the rotation of the system. This degree of freedom can take the energy from the surroundings and also put it back into the surroundings. Not only temperatures but also rotational states (rotational speeds) must therefore be taken into account in the description of the efficiency. We refer to the new type of heat engines proposed here as RS machines (machine operating on the basis of the rotating system).

With conventional heat engines, the thermal expansion or shrinkage of the working material (e.g. a gas) is directly converted into mechanical work. The new working principle

\footnotetext{
* Corresponding author: $\underline{\mathrm{m}-\mathrm{mesina} @ \mathrm{t} \text {-online.de }}$
}

Reviewers: Josef Soukup, Branislav Ftorek 
proposed here works differently. In the proposed concept, in the rotating systems the heat supply leads to the expansion of the working medium and thus to mass displacement in the direction of the rotational axis. This movement of the mass leads to a reduction of the inertia moment. This reduction results in the increase of the rotational frequency at a constant angular momentum and also an increase in the rotational energy. This phenomenon has long been used by ice skaters when performing pirouettes. However, the muscle work of the athletes causes a shift in mass. In an RS machine, the heat supplied is the cause of the mass shift.

The aim of the calculations carried out was to check whether this phenomenon was sufficient to increase efficiency beyond the Carnot limit. The functioning of the RS machine as described here allows the user - due to a partially selectable starting rotational frequency - to determine the amount of heat that can be converted to mechanical energy, at fixed working temperatures $\mathrm{T}_{1}$ and $\mathrm{T}_{2}$.

The RS machines operate cyclically, each cycle has 4 phases. In phase 1 , the system is operated at the ambient temperature $T_{1}$ and accelerated at the rotational speed $\omega_{1}$. In this phase, mechanical energy must be supplied to the system. In phase 2 , the temperature of the working liquid is increased from $T_{1}$ to $T_{2}$. To simplify our calculations, we have assumed that the necessary heat is supplied at a constant angular momentum. The rotational speed increases to $\omega_{2}$. In phase 3 , the rotational energy is removed from the system at the constant temperature $T_{2}$ and the system is decelerated. In phase 4 , the working liquid is cooled down to the $T_{1}$ temperature. The work medium could still work in phase 4 , but we have not included this to simplify the calculations.

When the rotation in phase 1 is accelerated, only in the case of a compressible liquid, some of the energy supplied is consumed to compress the liquid. In the following calculations, we assume that when the rotation is decelerated in phase 3 , the braking of the rotation leads to decompression and the decompression energy is converted into rotational energy.

\section{Description of the geometry of the RS machine (simplest version)}

The basic principle of an RS machine can be seen in figures 1-3. The main part of the machine is a hollow cylinder that is to rotate around its axis. The working fluid first has the $\mathrm{T}_{1}$ temperature and is in a cylindrical shell in the rotation with the outer radius $\mathrm{R}_{\mathrm{a}}$ and the internal radius $\mathrm{R}_{\mathrm{i} 1}$ (see Figure 1 and 2). The influence of gravitation on the geometry of the working fluid is not included.

In the container are at least two partition walls, which serve to ensure the rotation of the working medium together with the container. The partitions are mechanically fixed to the container. In addition, by pressure on the separating walls, the expanding liquid can change the rotational state of the system. The geometry described here has deliberately not been selected optimally! The article only intends to show that even with this simple and nonoptimal design, higher efficiency values can be achieved than according to Carnot. The greatest advantage of this geometry is that the computational check can be performed very easily. 


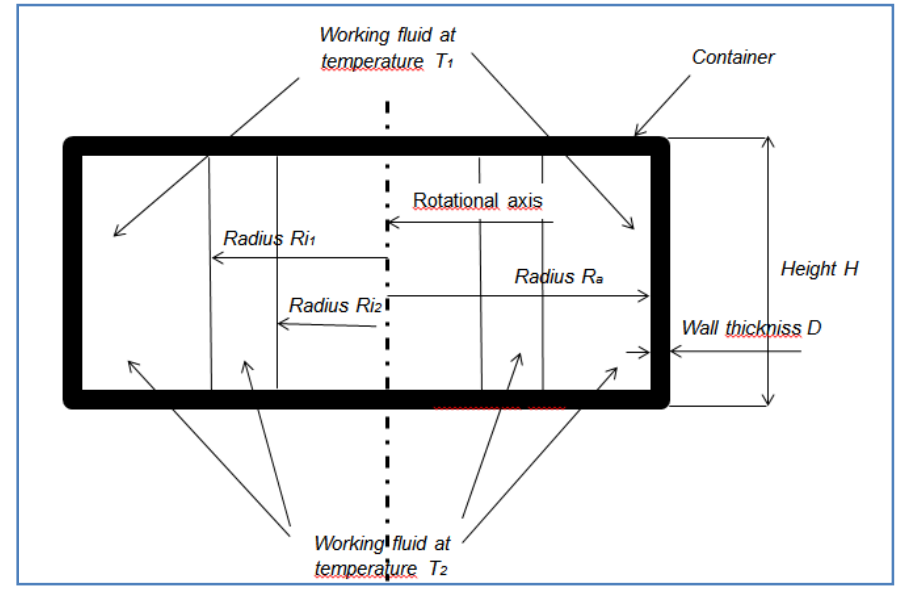

Fig. 1. RS machine, side view, perpendicular to the axis of rotation

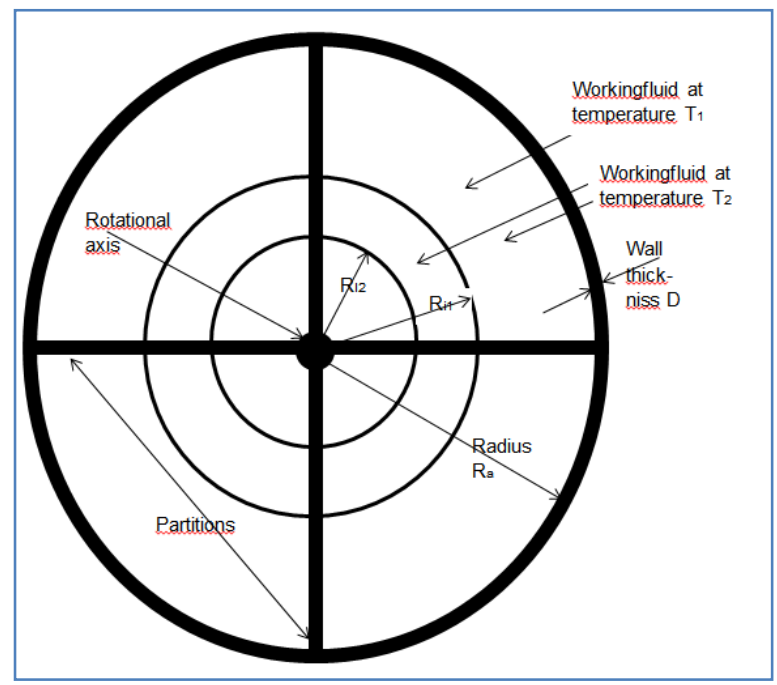

Fig. 2. Geometry of an RS machine, view from above, in the direction of the rotational axis

After heating to the temperature $T_{2}$ the liquid is in the cylinder with the outer radius Ra and in the inner radius $R_{i 2}$. Because $T_{2}>T_{1}$ (expansion in the direction of the axis of rotation), $\mathrm{R}_{\mathrm{i} 2}$ is also $<\mathrm{R}_{\mathrm{i} 1}$. This results in the reduction of the moment of inertia. For all further considerations, we assume that with the temperature transition from $T_{1}$ to $T_{2}$ the container geometry remains virtually unchanged. 


\section{Basic formulas for the efficiency calculations of the RS machine}

Terms:

$\mathrm{M}_{\mathrm{flu}}$ - Mass of the working medium,

$\rho\left(\mathrm{P}_{1}, \mathrm{~T}_{1}\right)$ - density of the liquid used at initial pressure $\mathrm{P}_{1}$ and temperature $\mathrm{T}_{1}$,

$\mathrm{C}_{\mathrm{vFlu}}$ - specific heat of the liquid,

$\mathrm{H}$ - Container hight,

$\mathrm{M}_{\text {Cont }}$ - mass of the container,

$\rho_{\text {Cont }}$ - density of the container material,

$\mathrm{Cv}_{\text {Cont }}$ - specific heat of the container material,

$\alpha$ - thermal expansion coefficient of the liquid,

$\mathrm{P}_{1}$ - initial pressure, $100000 \mathrm{~N} / \mathrm{m}^{2}$,

Working fluid - diethyl ether.

The geometry terms are described in the figures 1-3.

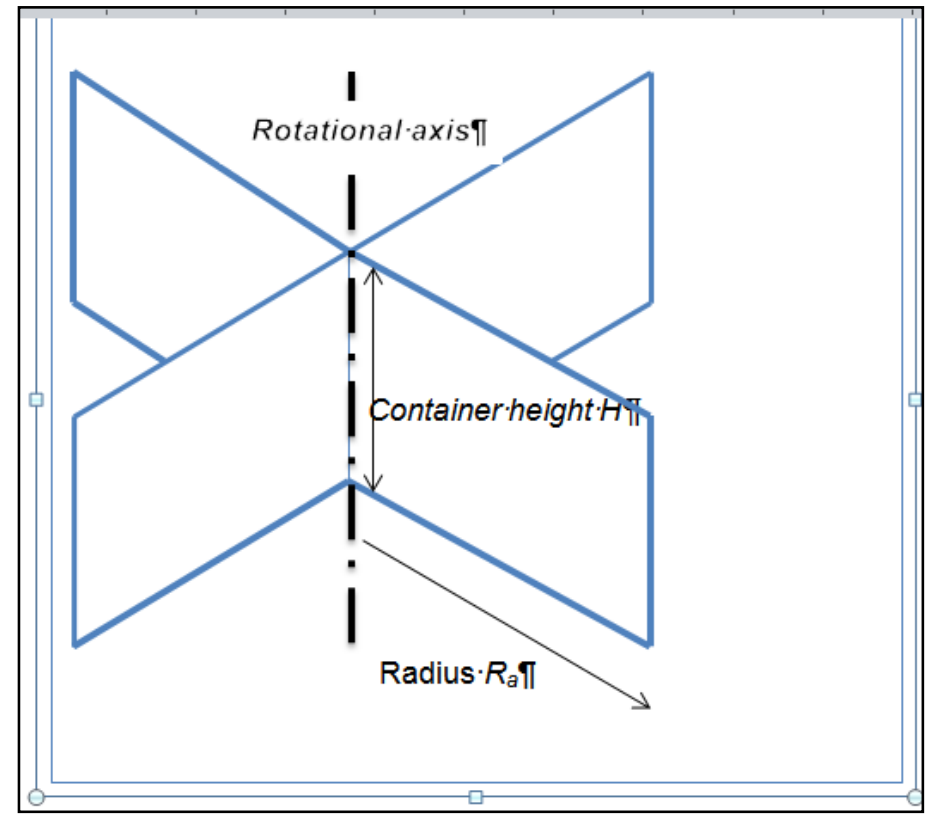

Fig. 3. Four partitions in the container of an RS machine

\section{Efficiency calculation for the incompressible medium}

At temperature $T_{1}$ and at pressure $P_{1}$ the following applies:

$$
M_{\mathrm{Flu}}=\pi *\left(R a^{2}-R i_{1}^{2}\right) * H * \rho\left(P_{1}, T_{1}\right)
$$

For incompressible media, we consider $\rho$ as dependent on temperature only.

The $\mathrm{M}_{\mathrm{Flu}}$ mass is located in the cylinder shell with the outer radius $\mathrm{Ra}$ and inner radius $\mathrm{Ri}_{1}$. Mass $\mathrm{M}_{\mathrm{Flu}}$ is equal to product volume $\left(\mathrm{P}_{1}, \mathrm{~T}_{1}\right)$ times the density $\left(\mathrm{P}_{1}, \mathrm{~T}_{1}\right)$. At temperature $\mathrm{T}_{2}$ the following applies: 


$$
M_{F l u}=\pi *\left(R a^{2}-R i_{2}^{2}\right) * H * \rho\left(P_{2}, T_{2}\right)
$$

The $\mathrm{M}_{\mathrm{Flu}}$ mass is located in the cylinder shell with the outer radius $\mathrm{Ra}$ and inner radius $\mathrm{Ri}_{2}$. From the equalisation of (1) and (2) the following applies:

$$
R i_{2}=\sqrt{R a^{2}-\left(R a^{2}-R i_{1}^{2}\right) * \frac{\rho\left(P_{1}, T_{1}\right)}{\rho\left(P_{2}, T_{2}\right)}}
$$

Since Ri2 $\geq 0$ must be, the temperature $T_{2}$ cannot be arbitrarily large for a given geometry. However, this limitation can be corrected by a small change in the geometry.

For the moment of inertia of a cylinder shell with the outer radius $\mathrm{Ra}$ inner radius $\mathrm{Ri}$, density $\rho$, and the height $\mathrm{H}$, the following applies:

$$
J(P, T)=\pi *\left(R a^{2}-R i^{2}\right) * H * \rho(P, T) * \frac{\left(R a^{2}+R i^{2}\right)}{2}
$$

In which $\rho$ is the material density of the liquid in the shell.

The formula (4) is used with various parameters for the calculations of the moments of inertia of the working fluid and the container.

We refer to $J_{1}$ as the complete moment of inertia of the whole system at the beginning of phase 2 and $J_{2}$ at the end of phase 2 .

$$
\begin{aligned}
& J_{1}=J_{1 F l u}+J_{\text {Cont }} \\
& J_{2}=J_{2 F l u}+J_{\text {Cont }}
\end{aligned}
$$

Assumption: The moment of inertia of the container $\mathrm{J}_{\text {Cont }}$ remains virtually unchanged in the temperature range used. The kinetic energy of rotation at the beginning of phase 2 is:

$$
E k_{1}=J_{1} * \frac{\omega_{1}^{2}}{2}
$$

At the end of phase 2

$$
E k_{2}=J_{2} * \frac{\omega_{2}^{2}}{2}
$$

Where $\omega=2 * \pi * f, f$ is the frequency of rotation. For the angular momentum $\mathrm{L}$ the following applies:

At the beginning of phase 2

$$
L_{1}=J_{1} * \omega_{1}
$$

At the end of phase 2

$$
L_{2}=J_{2} * \omega_{2}
$$

Ideally, the loss of rotational moment during phase 2 is 0 . In reality, there are losses in rotational momentum (eg due to friction). These losses can be represented by factor $\mathrm{K}_{\text {red }}$. 
Then:

$$
J_{2} \cdot \omega_{2}=J_{1} \cdot \omega_{1} * K_{\text {red }}
$$

Where $\mathrm{K}_{\mathrm{red}} \leq 1$ (the loss of the angular momentum). From the equation (11), the angular velocity $\omega_{2}$ at the end of phase 2 can be calculated:

$$
\omega_{2}=\left(\frac{J_{1}}{J_{2}}\right) * \omega_{1} * K_{r e d}
$$

For the kinetic energies $\mathrm{E}_{\mathrm{kin} 1}$ and $\mathrm{E}_{\mathrm{kin} 2}$ the following applies:

$$
E_{k_{2}}=E_{k_{1}} * \frac{J_{1}}{J_{2}} * K_{r e d}^{2}
$$

When $\mathrm{J}_{1} * \mathrm{~K}_{\text {red }}^{2}>\mathrm{J}_{2}$, then $\mathrm{E}_{\mathrm{k} 2}$ is also $>\mathrm{E}_{\mathrm{k} 1}$. This means an increase of the rotational energy due to the heat supply.

In the course of phase 3, the system can provide the kinetic energy $E_{\mathrm{k} 2}$. In phase 4, the working liquid and the container are cooled from temperature $T_{2}$ to temperature $T_{1}$. In order to simplify the calculations, at the end of phase 3 the remaining temperature difference between $T_{2}$ and $T_{1}$ are deliberately not used for the production of the work! Nevertheless, the achieved efficiency values can exceed Carnot's limit.

For the obtained mechanical energy delta $\mathrm{Ek}_{\mathrm{Ek}}$ the following applies:

$$
\operatorname{delta}_{E k}=E k_{2}-E k_{1}
$$

From equations (7-14) the following applies:

$$
\left.\operatorname{delta}_{E k}=\left(\left(\frac{J_{1}}{J_{2}}\right) * K_{r e d}{ }^{2}-1\right)\right) * E k_{1}
$$

For incompressible working media, we assume that the moments of inertia $\mathrm{J}_{1}$ and $\mathrm{J}_{2}$ are only dependent on temperatures $\mathrm{T}_{1}$ and $\mathrm{T}_{2}$.

Equation (15) shows that the gained energy delta $\mathrm{Ek}_{\mathrm{Ek}}$ is proportional with the kinetic energy $E_{1}$. As $\omega_{1}$ and thereby also $E_{k 1}$ can be selected freely, the efficiency can be influenced at least in part (see figures 5-8 and tables 2-5). This is a new and the most important property of RS machine, which leads to the possibility to exceed the Carnot limit of efficiency.

\section{Calculation of the efficiency $\eta$}

The efficiency of the above-described working cycle $\eta$ is defined as the work obtained in the cycle divided by the total amount of the heat supplied to the system.

The heat supplied must provide the following three tasks for incompressible working fluid) and four tasks for compressible liquid energy:

A1. Increase of the rotational energy by delta $\mathrm{Ek}_{\mathrm{Ek}}$, (equation 15). The increase of the kinetical energy delta $_{\mathrm{EK}}$ is the gained work in one working cycle (later the phase 2).

A2. Heating the working fluid (see Eq. 16) and the container see eq. (17) from temperature $T_{1}$ to temperature $T_{2}$. 
For the required heat quantity, the following applies:

$$
\begin{gathered}
D Q_{\text {Cont }}=M_{\text {Cont }} * C_{V \text { Cont }} *\left(T_{2}-T_{1}\right) \\
D Q_{F l u}=M_{F l u} * C_{V F l u} *\left(T_{2}-T_{1}\right)
\end{gathered}
$$

A3. As a result of the thermal expansion, some of the working liquid is displaced in the direction of the axis of rotation.

The necessary work $A_{\text {transl }}$ serves to overcome the centrifugal force. This work can be estimated from the following formula:

$$
A_{\text {transl }} \approx M_{F l u} * \frac{\alpha *\left(T_{2}-T_{1}\right)}{1+\alpha *\left(T_{2}-T_{1}\right)} \omega_{2}^{2}\left(R i_{1}-R i_{2}\right) * \frac{\left(R i_{1}+R i_{2}\right)}{2}
$$

Where $\mathrm{Ri}_{1}$ internal limit of the zone at temperature $\mathrm{T} 1, \mathrm{Ri}_{2}$ the internal limit at temperature $T_{2}$ and $M_{\text {Flüs }}$ is the mass of the working fluid in the zone (see Fig.1 and 2). For the calculation of the efficiency $\eta$ we make the pessimistic assumption that $A_{\text {transl }}$ can only be taken into account when calculating the heat supplied to the system. In our calculation, this work does not contribute to the energy that can be supplied by the system.

A4. For compressible fluids there is a $4^{\text {th }}$ task. In phase 3 for isothermal deceleration of rotation, the heat must also supply the expansion energy of the compressible medium. This task is not applicable for incompressible working media.

For the efficiency $\eta$ of the RS machine with incompressible working fluid the following applies:

$$
\eta=\frac{\operatorname{delta}_{k}}{\operatorname{deltaE}_{k}+D Q_{\text {Kont }}+D Q_{\text {Flu }}+A_{\text {transl }}}
$$

Formulas (14) - (19) show that the efficiency is dependent not only on the temperatures $T_{1}$ and $T_{2}$, but also on the angular velocity $\omega_{1}$ or frequency $F_{1}$ as well as the material data. By the practical application of formulas (4) - (19), it can be easily shown (s. figure 4) for many combinations of $\omega_{1}, T_{1}$, and $T_{2}$, that the efficiency $\eta$ is greater than the Carnot limit.

Because the gained energy delta $\mathrm{Ek}_{\mathrm{k}}$ and the work $\mathrm{A}_{\text {transl }}$ are quadratically dependent on frequency $F_{1}$, converges with increasing frequency $F_{1}$ to a value that is less than 1 , but can still be greater than Carnot's. The real liquids are compressible. Formula (19) can therefore only be used for rough estimates of the efficiency values.

Formula (19) has to be modified for calculating the efficiency.

Our calculations for compressible fluids are based on the following formula:

$$
\rho\left(P_{2}, T_{2}\right)=\rho\left(P_{1}, T_{1}\right) \frac{\left(1+\kappa\left(P_{2}-P_{1}\right)\right)}{\left(1+\alpha\left(T_{2}-T_{1}\right)\right)}
$$

$P_{1}, P_{2}$ - different pressure values,

$T_{1}, T_{2}$ - different temperatures,

$\rho$ - pressure and temperature dependent density,

$\alpha$ - thermal expansion coefficient,

$\varkappa$-Compressibility coefficient.

Assumption: The parameters $\alpha$ and $x$ are considered constant. Formula (20) can be seen as a substitute for the state equation of the liquid. 
In order to calculate the location dependency of the density, we divided the total volume of the liquids into $\mathrm{N}$ sub-regions (cylinder shells) of the same mass. In our test examples it was found sufficient to take $\mathrm{N}=30$ and 40 radial zones, respectively. When the temperature or $\omega$ changes, only the boundaries between the subareas shift. Pressure in the radial zones is location-dependent and increases in the direction of the outer edge by the own weight of the liquid in the radial zone.

For the efficiency calculation the following applies:

$$
\eta=\frac{E_{\text {dekompr }}-E_{\text {kompr }}+\text { deltaEk }}{\text { deltaEk }+D Q_{\text {Kont }}+D Q_{F l u}+A_{\text {transl }}+E_{\text {dekompr }}}
$$

Let us call $\mathrm{E}_{\mathrm{kompr}}$ the necessary energy for the compression of the liquid in the phase 1 and as $E_{\text {dekompr }}$ the releasing energy in the expansion of the liquid in phase 3 . In the counter of the fraction in equation (21) is the total energy that can be taken from the system in one cycle. In the denominator is the total heat required to be supplied to the system in one cycle.

\section{Advantages of the RS machine:}

- Efficiency is much better in many situations than according to Carnot

- Numerous designs and optimisation options are possible (e.g. geometry, material selection, rotational speed)

- Use of small temperature differences

- Regeneration possibility of the used heat reservoir by the heat supply from the environment

- In principle, a new energy source (as perpetuum mobile of 2nd type)

- A technically possible use of the water-ice anomaly. When freezing water, the water can only expand in the direction of the rotational axis.

\section{Disadvantages of the RS machine:}

- Very high rotational speeds are necessary (see figures 4 and 5), but possible in the context of today's technology.

- Isothermal acceleration (phase 1) and deceleration of the rotation (phase 3) may be very slow.

- Special materials recommended (maximum thermal expansion and low compressibility)

- Other operation than with conventional heat engines - isothermal acceleration of rotation at temperature $T_{1}$ (phase 1) and isothermal deceleration of the rotation at temperature $\mathrm{T}_{2}$ in phase 3 .

- Heat supply with an angular momentum as constant as possible (phase 2)

- In order to simplify the calculations, we assume that in phase 4 the working fluid is cooled from temperature $T_{2}$ to temperature $T_{1}$ without performing work.

\section{Further development possibilities:}

- The use of the phase change (liquid gas, solid phase gas)

- Use of residual heat at the end of phase 3

- Heating the working fluid without heating the whole container

- Optimising the geometry 


\section{Proposal for verifying the theory}

The container with the working fluid must be accelerated at temperature $T_{1}$ to the angular velocity $\omega_{1}$. The necessary energy $E_{1}$ for this is measured. Then the system must be heated to temperature $T_{2}$ at a constant angular momentum. We call the heat necessary for this $\mathrm{Q}_{2}$. After this in phase 3, the rotation is completely decelerated to $\omega=0$ at a constant temperature $T_{2}$. The energy $E_{3}$ with drawn from this and the amount of $Q_{3}$ supplied is measured.

The heat $\mathrm{Q}_{2}$ and $\mathrm{Q}_{3}$ should be obtained from a heat reservoir with the temperature $\mathrm{T}_{2}$. For test purposes only, the required amount of heat could be generated electrically or chemically directly in the system.

The efficiency can be determined from the measured data (see equation (22)).

$$
\eta=\frac{E_{3}-E_{1}}{Q_{2}+Q_{3}}
$$

The internal structure of the RS machine does not have to be known for measuring the efficiency!

\section{Some computed results}

Table 1. Common input data for all test calculations

\begin{tabular}{|c|c|}
\hline Working fluid: & diethyl ether \\
\hline Density: & $738 \mathrm{~kg} \mathrm{~m}^{3}$ at 273 degrees $\mathrm{K}$. \\
\hline Compressibility $\boldsymbol{x}$ & $1.84 \mathrm{E}-09 / \mathrm{GPa}$ \\
\hline Thermal expansion coefficient $\boldsymbol{\alpha}$ & $0.00162 / \mathrm{deg} \mathrm{K}$ \\
\hline Specific heat of the liquid Cv: & $2310 \mathrm{~J} /(\mathrm{Kg}$. degree $\mathrm{K})$ \\
\hline Material of container: & aluminium \\
\hline Density: & $2700 \mathrm{Kg} / \mathrm{m}^{3}$ \\
\hline Specific heat $\mathbf{C v}_{\text {cont }}$ & $896 \mathrm{~J} /(\mathrm{Kg} \cdot$ degree $\mathrm{K})$ \\
\hline
\end{tabular}

Other data for the calculation: $\mathrm{Ra}=0.5 ; \mathrm{H}=0.4 ; \mathrm{D}=0.015$. 


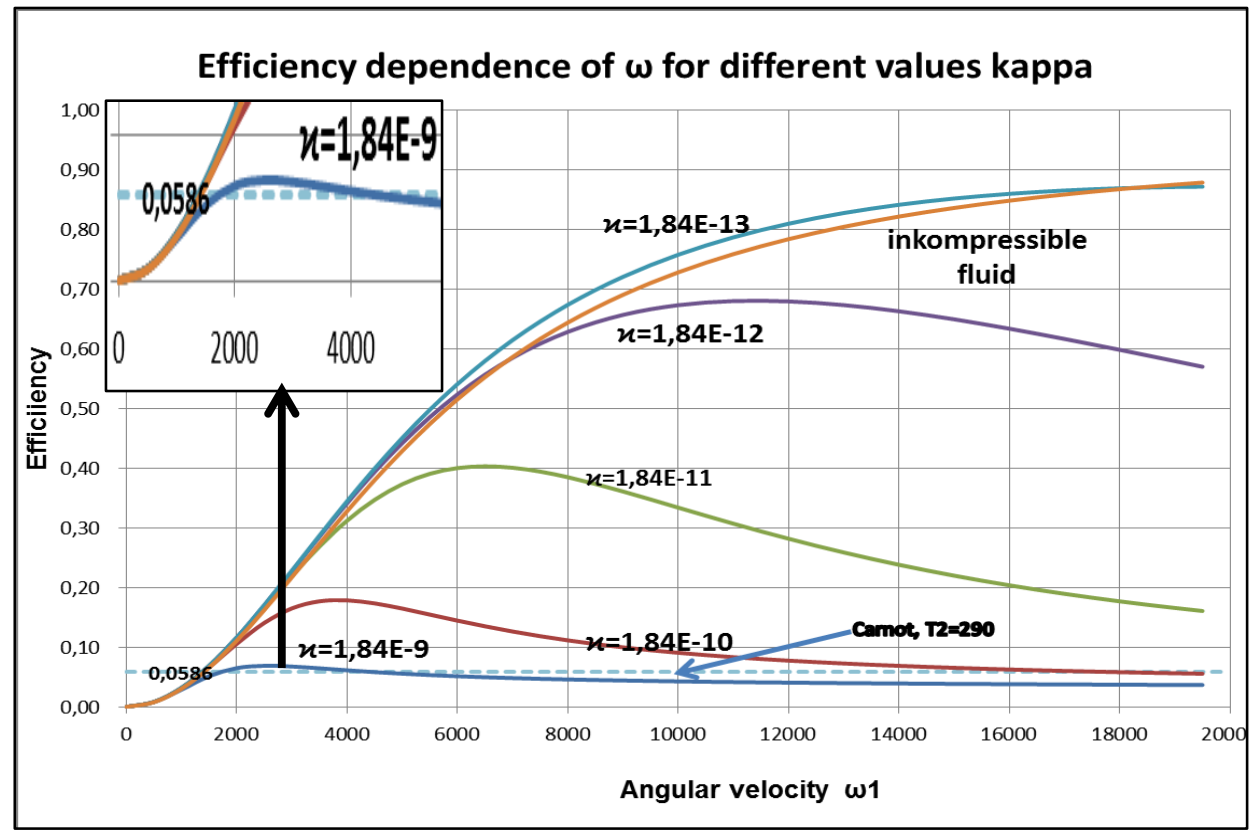

Fig. 4. Efficience dependence from $\omega_{1}$ for various coefficients of compressibility and for incompressible fluid, $\mathrm{T}_{2}=290$

Input data for calculation: $\mathrm{Ra}=0,5 ; \mathrm{H}=0,4 ; \mathrm{D}=0,015 ; \mathrm{T}_{2}=290$; various values of kappa.

In Fig. 4 and 5 Carnot's limits were drawn as horizontal lines (horizontal means independent from $\left.\omega_{1}\right)$. The maximum of the efficiency curve can be clearly above the Carnot's limits (e.g. for the temperatures T2 $=275$ and $290 \mathrm{~K}$ ). For all $\omega_{1}$ from interval [UGR, OGR], the calculated efficiency is greater than the Carnot's limit.

Table 2. Some important parameters of $\omega_{1}$-dependence curves of efficiency, from figure 5

\begin{tabular}{|c|c|c|c|c|}
\hline Temperature $\mathbf{T}_{\mathbf{2}}$ & 320 & 300 & 290 & 275 \\
\hline Eta Carnot & 0,146875 & 0,09 & 0,058621 & 0,007273 \\
\hline Eta RS machine max. value & 0,11720 & 0,08758 & 0,06882 & 0,02296 \\
\hline Eta max at $\boldsymbol{\omega} \mathbf{1}$ approximately & 4076 & 3100 & 2575 & 1275 \\
\hline Angular speed $\boldsymbol{\omega}_{\mathbf{1}}, \mathbf{U G R}$ & no solution & no solution & 1700 & 475 \\
\hline Angular speed $\boldsymbol{\omega}_{\mathbf{1}}, \mathbf{O G R}$ & no solution & no solution & 4400 & 5550 \\
\hline
\end{tabular}

Input data for the calculation: $\mathrm{Ra}=0.5 ; \mathrm{H}=0.4 ; \mathrm{D}=0.015 ;$ kappa $=1.84 \mathrm{E}-09$ 


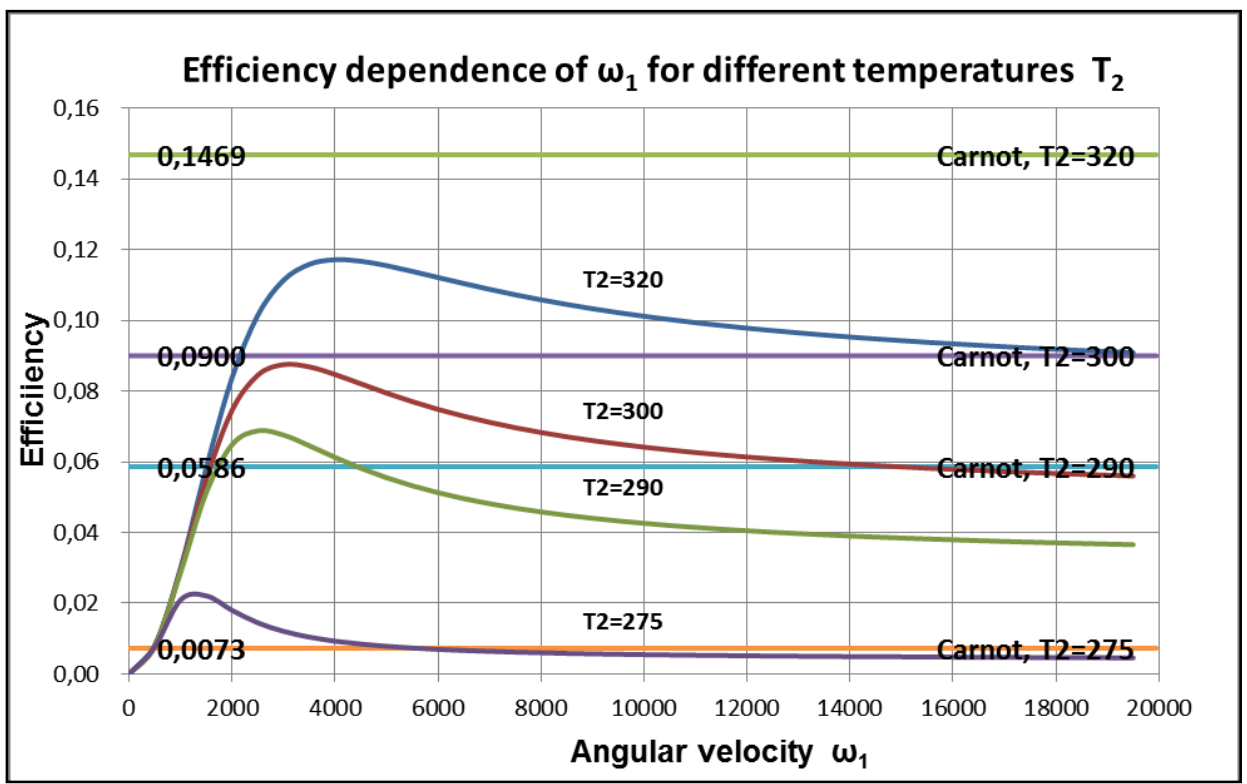

Fig. 5. Efficiency dependence of $\omega_{1}$ for different temperatures $T_{2}$, comparison with Carnot's limit

Input data for the calculation: $\mathrm{Ra}=0.5 \mathrm{~m} ; \mathrm{H}=0.4 \mathrm{~m} ; \mathrm{D}=0.015 \mathrm{~m} ; \mathrm{Kappa}=1.84 \mathrm{E}-09$.

Figures 4-5 show that the efficiency of an RS machine increases as a function of the angular velocity $\omega_{1}$ up to a maximum value. Reducing it after achieving maximum efficiency is thereby explained because the difference $\left(E_{\text {dekompr }}-E_{\text {kompr }}\right)$ increases in comparison to the energy growth in phase 2 . For the increasing rotational frequency $\omega_{1}$, the $\mathrm{RS}$ machine is more similar to conventional heat engines in terms of efficiency.

At the temperature $\mathrm{T} 2=320$ and $\mathrm{T} 2=300 \mathrm{~K}$ (s. the figure 5), the Carnot limit cannot be exceeded only by the change of $\omega_{1}$, at constant value of other selected parameters $R_{a}, M_{\text {Flu }}$, height $\mathrm{H}$ and wall thickness of the container $\mathrm{D}$.

Exceeding the Carnot's limit is easier at low temperature $T_{2}$ (at low Carnot's limit) than at higher temperature $\mathrm{T}_{2}$ (see. figure 5). 


\section{Conclusion}

What makes it possible to say that even heat engines with a better efficiency than Carnot are possible?

In the patent application, the basic principle of RS machine has been presented. The efficiency of this machine can be described through formulas, which are transparent, trivial, and comprehensible. It is an attempt to simulate the working cycle of the RS machine. The simulation of the processes in nature is now a standard tool in natural science and in technology.

The most important feature of this simulation is that the efficiency of the RS machine is explicitly dependent on the rotational speed $\omega$. It is by no means possible to eliminate the parameter $\omega$. The calculations carried out show that the calculated efficiency can clearly exceed the Carnot's limit in many situations.

In combination with an ideal heat pump, it is possible to extract heat from the surroundings and convert it into mechanical work.

I am particularly grateful to J. Pimiskern for many helpful discussions, for many interesting references to this topic, and for his help in the design of this publication.

\section{References}

1. Patent application DE 102011104914 A1

2. H. Kuchling, Taschenbuch der Physik. $\left(20^{\text {th }}\right.$ Edition, Carl Hanser Verlag Leipzig, 2011), ISBN 978-3-446-42457-9 